\title{
Le bibliothécaire québécois : d'un homme de lettres à un professionnel de l'information
}

\section{The Québec Librarian: From a Man of Letters to an Information Professional \\ El bibliotecario quebequense: de un hombre de letras a un profesional de la información}

\section{Marcel Lajeunesse}

Volume 51, numéro 2, avril-juin 2005

Les métiers du livre au Québec

URI : https://id.erudit.org/iderudit/1030095ar

DOI : https://doi.org/10.7202/1030095ar

Aller au sommaire du numéro

Éditeur(s)

Association pour l'avancement des sciences et des techniques de la documentation (ASTED)

ISSN

0315-2340 (imprimé)

2291-8949 (numérique)

Découvrir la revue

Citer cet article

Lajeunesse, M. (2005). Le bibliothécaire québécois : d'un homme de lettres à un professionnel de l'information. Documentation et bibliothèques, 51(2), 139-148. https://doi.org/10.7202/1030095ar
Résumé de l'article

Les Romain, père et fils, furent connus au tournant des années 1800 comme les premiers bibliothécaires du Québec, tant à la Bibliothèque de Québec qu'à celle de la nouvelle Assemblée législative. Jusque vers 1930, les bibliothécaires étaient des écrivains, des hommes de lettres sans formation spécialisée. Il en fut ainsi pour Étienne Parent, Pamphile LeMay, Narcisse-Eutrope Dionne, Aegidius Fauteux, Hector Garneau et Pierre Boucher de Crèvecoeur. Au cours des années 1930-1960, on constate une structuration de la profession, avec l'agrément par l'American Library Association (ALA) de l'École de McGill (1928), de la fondation de l'École de bibliothécaires (1937) de la Quebec Library Association (1932) et d'une association francophone (1943). Les années 1960 apportent des changements majeurs dans la bibliothéconomie québécoise. L’Assemblée législative vote, en décembre 1959, la première loi sur les bibliothèques publiques. Les collèges classiques se dotent en ces années de véritables bibliothèques. En raison du développement des études supérieures et de la recherche, les universités ont besoin de bibliothèques beaucoup mieux pourvues. L'École de bibliothéconomie de l’Université de Montréal est créée en 1961. Les bibliothécaires eux-mêmes, diplômés d’université, privilégient dorénavant une vision scientifique de leur profession. Ils mettent sur pied en 1969 la Corporation des bibliothécaires professionnels du Québec.
Tous droits réservés (c) Association pour l'avancement des sciences et des techniques de la documentation (ASTED), 2005
Ce document est protégé par la loi sur le droit d'auteur. L’utilisation des services d’Érudit (y compris la reproduction) est assujettie à sa politique d'utilisation que vous pouvez consulter en ligne.

https://apropos.erudit.org/fr/usagers/politique-dutilisation/ 


\title{
Le bibliothécaire québécois: d'un homme de lettres à un professionnel de l'information
}

\author{
MARCEL LAJEUNESSE \\ École de bibliothéconomie et des sciences de l'information \\ Université de Montréal \\ marcel.lajeunesse@umontreal.ca
}

\begin{abstract}
RÉSUMÉ | ABSTRACTS | RESUMEN
Les Romain, père et fils, furent connus au tournant des années 1800 comme les premiers bibliothécaires du Québec, tant à la Bibliothèque de Québec quà celle de la nouvelle Assemblée législative. Jusque vers 1930, les bibliothécaires étaient des écrivains, des hommes de lettres sans formation spécialisée. Il en fut ainsi pour Étienne Parent, Pamphile LeMay, NarcisseEutrope Dionne, Egidius Fauteux, Hector Garneau et Pierre Boucher de Crèvecoeur. Au cours des années 1930-196o, on constate une structuration de la profession, avec l'agrément par l'American Library Association (ALA) de l'École de McGill (1928), de la fondation de l'École de bibliothécaires (1937), de la Quebec Library Association (1932) et d'une association francophone (1943). Les années 1960 apportent des changements majeurs dans la bibliothéconomie québécoise. L'Assemblée législative vote, en décembre 1959, la première loi sur les bibliothèques publiques. Les collèges classiques se dotent en ces années de véritables bibliothèques. En raison du développement des études supérieures et de la recherche, les universités ont besoin de bibliothèques beaucoup mieux pourvues. L'École de bibliothéconomie de l'Université de Montréal est créée en 1961. Les bibliothécaires eux-mêmes, diplômés d'université, privilégient dorénavant une vision scientifique de leur profession. Ils mettent sur pied en 1969 la Corporation des bibliothécaires professionnels du Québec.
\end{abstract}

\section{The Québec Librarian: From a Man of Letters to an Information Professional}

The Romains, both father and son, were known at the turn of the $19^{\text {th }}$ century as the first librarians in Québec, both at the Bibliothèque de Québec as well as the new library of the Legislative Assembly. Up until about 1930, librarians were authors, literary men without any special training. Such was the case of Etienne Parent, Pamphile LeMay, Narcisse-Eutrope Dionne, Egidius Fauteux, Hector Garneau and Pierre Boucher de Crèvecour. During the period from 1930 to 1960 , one observes the formal organisation of the profession with the accreditation of the McGill School by the American Library Association (ALA) in 1928, and the creation of the École des bibliothécaires (1937), the Québec Library Association (1932) and of a francophone association (1943). The 196os brought major changes to librarianship in Québec. In December 1959, the Legislative Assembly adopted the first public libraries act. Classical colleges established libraries. With the development of higher education and research, university libraries realised that they had to become better equipped. In 1961, the École de bibliothéconomie of the Université de Montréal was founded. University-trained librarians have adopted a more scientific approach to their profession. In 1969, they created the Corporation of Professional Librarians of Québec.
El bibliotecario quebequense: de un hombre de letras a un profesional de la información

Los Romain, padre e hijo, fueron conocidos alrededor de 1800 como los primeros bibliotecarios de Quebec, en la Biblioteca de Quebec y en la de la nueva Asamblea Legislativa. Hasta 1930, los bibliotecarios fueron escritores, hombres de letras que carecian de formación especializada. Tal fue el caso de Étienne Parent, Pamphile LeMay, Narcisse-Eutrope Dionne, Agidius Fauteux, Hector Garneau y Pierre Boucher de Crèvecoeur. Durante los años 1930 a 1960, se constata una estructuración de la profesión, con la acreditación de la American Library Association (Asociación Americana de Bibliotecas -- ALA) de la Escuela de Biblioteconomía de la Universidad McGill (1928), la fundación de la Escuela de Bibliotecarios (1937), la Quebec Library Association (Asociación Quebequense de Bibliotecas,1932) y de una asociación similar de habla francesa (1943). La década de los años 60 aporta cambios importantes a la biblioteconomía quebequense. La Asamblea Legislativa vota, en diciembre de 1959, la primera ley sobre las bibliotecas públicas. En estos años los colegios secundarios clásicos se dotan de verdaderas bibliotecas. Debido al desarrollo de los estudios superiores y de la investigación, las universidades tienen necesidad de bibliotecas mucho más completas. La Escuela de Biblioteconomía de la Universidad de Montreal se creó en 1961. A partir de entonces, los bibliotecarios mismos, diplomados universitarios, dan prioridad a una visión científica de su profesión. En 1969 crean la Corporación de Bibliotecarios Profesionales de Quebec.

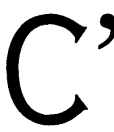

EST UN LIEU COMmUn d'AfFirmer qu'il y a des bibliothécaires depuis qu'il y a des bibliothèques. On a créé et établi des bibliothèques depuis l'Antiquité, depuis que l'homme produit des écrits. Or, l'écrit a précédé le livre. Les civilisations du Tigre, de l'Euphrate et du Nil ont vu naitre les premières bibliothèques et les premiers bibliothécaires.

Dans cette partie du Nord de l'Amérique, les livres et les bibliothèques ont accompagné les colonisateurs français, issus eux-mêmes de cette civilisation du livre développée en métropole. Le premier réflexe de Cartier, de Champlain, de Boucher et des Jésuites est de publier des relations de leurs voyages, de leurs explorations, de leurs expériences missionnaires et aussi des richesses naturelles de la nouvelle colonie. De la Nouvelle-France, nous connaissons deux types de bibliothèques: la bibliothèque privée de curé, de 


\section{La première bibliothèque canadienne qui a marqué l'histoire des bibliothèques d'ici est sans conteste la Bibliothèque du Collège des Jésuites de Québec.}

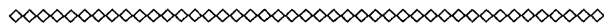

seigneur, d'entrepreneur et d'administrateur colonial, et la bibliothèque collective des Sulpiciens montréalais, du Séminaire de Québec, du Collège des Jésuites et du Conseil souverain (Lajeunesse, 2004). Ces bibliothèques reflètent bien l'esprit de la Contre-Réforme catholique et l'humanisme du Siècle des lumières naissant.

L'histoire des bibliothèques s'est intéressée aux institutions, mais elle a négligé celle des bibliothécaires. En France, le Centre de recherche en histoire du livre a tenu, en 2003, un colloque sur le sujet dans une "perspective de l'histoire comparée [afin] de favoriser une vision plus globale de l'histoire des bibliothécaires, en France comme dans d'autres aires géographiques" (Moledina, 2004). Frédéric Barbier a dégagé trois axes autour desquels ce champ d'étude pouvait s'articuler: le statut social et administratif du bibliothécaire, les fonctions matérielles et culturelles liées à cette charge, et enfin le rôle du bibliothécaire. Il importe de situer le bibliothécaire parmi les métiers du livre et de décrire lévolution de son statut, de sa fonction et de son rôle. Gilles Gallichan avait, pour sa part, posé la question en 1988, en s'interrogeant sur «les bibliothécaires et leur conscience historique» et en évoquant la méconnaissance des pionniers de la bibliothéconomie en notre milieu. Il constatait par ailleurs que l'informatique n'était que l'un des nombreux changements vécus par les bibliothécaires dans l'exercice de leur profession au fil de l'histoire (Gallichan, 1988).

\section{DU BIBLIOTHÉCAIRE HOMME DE LETTRES}

La première bibliothèque canadienne qui a marqué l'histoire des bibliothèques d'ici est sans conteste la Bibliothèque du Collège des Jésuites de Québec. En 1972, à l'occasion de l'Année internationale du livre, la Bibliothèque nationale du Canada avait organisé une exposition sur la plus importante bibliothèque de la Nouvelle-France et André Beaulieu avait publié un catalogue de 138 volumes retenus à cette occasion (Beaulieu, 1972). On n'a de cesse de reconstituer le catalogue de cette bibliothèque dispersée au cours du dernier quart du XVIII ${ }^{\mathrm{e}}$ siècle: Antonio Drolet (1961) a recensé 622 volumes de cette bibliothèque dans la région de Québec, Claude Pariseau (1972) en a ajouté 66 provenant des bibliothèques des Sulpiciens et de la Bibliothèque nationale du Québec, et enfin Paul-Émile Filion (1977) en a retrouvé 62 autres dans la Bibliothèque jésuite de l'Immaculée-Conception, cette dernière ayant hérité des livres de l'abbé Nazaire Dubois provenant de John Neilson. Nous connaissons peu de chose sur les bibliothécaires de cette institution. Nous savons seulement que le catalogue des obédiences de la Compagnie de Jésus indique pour l'année 1716 le père Pierre Laure comme responsable de la bibliothèque (Drolet, 1961) et que le père Laure était aussi professeur dans les classes de grammaire, d'humanités et de rhétorique de ce collège (Audet, 1971). Il est permis de penser que le catalogue de 1720 est fort probablement le fruit du travail du régent Pierre Laure (Beaulieu, 1972). Il semble qu'au cours $\mathrm{du} \mathrm{XVIII}^{\mathrm{e}}$ siècle la bibliothèque fut à la charge d'un assistant du supérieur du Collège des Jésuites.

La connaissance que nous avons de la Bibliothèque du Séminaire de Québec à la fin du XVIII ${ }^{\mathrm{e}}$ siècle provient du catalogue qu'en a dressé l'abbé Arnaud Dudevant en 1782. Venant de Bordeaux, l'abbé Dudevant débarque à Québec en 1775, est agrégé au Séminaire de Québec en avril 1777 et y est ordonné prêtre le même mois. Il démissionne de ses fonctions de premier assistant du supérieur en $\mathbf{1 7 8 2}$ et procède à l'inventaire de la Bibliothèque du Séminaire avant de rentrer en France en 1783. Le catalogue montre que cette bibliothèque est de loin la plus importante au Canada, avec ses 4883 volumes et 2121 titres. Similaire à celles des établissements d'enseignement de France, elle comporte des sections consacrées à la théologie et à la religion, au droit civil et canonique, à l'histoire, aux belles-lettres et aux livres de classe. Le tiers des livres sont en latin. On trouve parmi eux les grands auteurs des $\mathrm{XVII}^{\mathrm{e}}$ et $\mathrm{XVIII}{ }^{\mathrm{e}}$ siècles, tel Voltaire avec son Dictionnaire philosophique portatif et Le Siècle de Louis XIV (Galarneau, 1980).

La Conquête britannique apporte au Bas-Canada deux nouveaux types de bibliothèques: d'abord, une bibliothèque "publique» de souscription, fondée à l'initiative du gouverneur Frederick Haldimand en 1779, puis, au début du XIX ${ }^{\mathrm{e}}$ siècle, une bibliothèque parlementaire, à la suite de la mise en place de la Chambre d'assemblée du Bas-Canada. François Romain fut le premier directeur de la Bibliothèque du Québec. Il dirigea également la Bibliothèque du Parlement, où son fils François Romain lui succéda, bien que les responsables de la Bibliothèque du Parlement eussent été les greffiers de la Chambre Samuel Phillip de $\mathbf{1 8 0 2}$ à 1808 et William Lindsay de 1808 à 1829 (Thériault, 1987; Gallichan, 1991). Les Romain peuvent être considérés comme les premiers Canadiens à avoir exercé la profession de bibliothécaire au Bas-Canada.

Depuis le début du XIX ${ }^{\mathrm{e}}$ siècle, la Bibliothèque du Parlement, à Québec, a attiré des bibliothécaires qui ont marqué la profession. Étienne Parent en fut responsable de 1833 à 1835 , deux années au cours desquelles il réorganisa la collection, réforma les règlements et assura un plus grand accès à la Bibliothèque. Il voulait 
ainsi doter le Bas-Canada d'une bibliothèque nationale qui, à l'instar de celle des États-Unis, serait ouverte à tous les citoyens. À l'emploi de la Bibliothèque législative du Haut-Canada depuis 1835, Alpheus Todd devint, sous l'Union, responsable de la Bibliothèque du Parlement de 1856 à 1867, avant de devenir bibliothécaire du nouveau Parlement fédéral à Ottawa en 1867 et l'une des sommités en droit constitutionnel au Canada. Il fut l'artisan de la reconstruction de la Bibliothèque parlementaire après les incendies de 1849 et de 1854 (Falardeau, 1972; Gallichan, 1991).

Il faut accorder une place particulière à GeorgesBarthélemi Faribault, qui fut le premier bibliographe $\mathrm{du}$ Bas-Canada. Entré dans la fonction publique en 1812, à 22 ans, responsable dans la décennie 1830 des archives parlementaires, traducteur et bibliophile, il poursuivit, avec l'appui d'Étienne Parent, ses travaux bibliographiques sur l'histoire de l'Amérique en vue de créer une collection nationale. Encouragé par la suite par les bibliothécaires Jasper Brewer et William Winder, Faribault ne cessa d'enrichir les collections de la Bibliothèque du Parlement en dépit des deux incendies de 1849 et de 1854 . Son Catalogue d'ouvrages sur l'histoire de l'Amérique (1837) est une œuvre capitale dans le développement de l'intérêt pour l'histoire nationale au XIX ${ }^{\mathrm{e}}$ siècle et pour l'histoire de la bibliographie canadienne.

En 1867, Pierre-Joseph-Olivier Chauveau, nommé premier ministre de la nouvelle province du Québec, soucieux d'encourager les jeunes écrivains prometteurs, offrit à Pamphile LeMay, avocat, traducteur, romancier et poète, le poste de bibliothécaire de l'Assemblée législative du Québec. Le geste de Chauveau s'inspirait de la tradition française d'offrir un poste de bibliothécaire à des hommes de lettres, comme ce fut le cas pour Sainte-Beuve à la Mazarine, dans la décennie 1840, et Leconte de Lisle, au Sénat en 1872, soit peu après qu'il ait refusé un poste similaire offert par Napoléon III sous le Second Empire. Cette tradition de sinécures va se perpétuer au $\mathrm{XX}^{\mathrm{e}}$ siècle avec les nominations de Paul Morin à la bibliothèque de l'École des beaux-arts de Montréal dans la décennie 1920 et de Robert Choquette, qui lui succédera en 1928 (Morin, 200o; Choquette, 1959).

La fonction que remplit Pamphile LeMay pendant 25 ans, de 1867 à 1892, ne fut toutefois pas une sinécure. Il eut à reconstruire la bibliothèque parlementaire à Québec, à intégrer la Bibliothèque de l'éducation, formée par Chauveau à partir de 1855, à celle du ministère de l'Instruction publique en 1875 et à vivre l'incendie de 1883 , qui détruisit une grande partie de sa bibliothèque. LeMay constitua plusieurs catalogues (1869, 1873 avec suppléments, 1879, 1884), dont la première version sur fiches. À son départ en 1892, la bibliothèque parlementaire, ouverte au grand public, comptait 33804 volumes (Pellerin, 1998). Sa tâche de responsable de la bibliothèque n'était pas
[Le] Catalogue d'ouvrages sur l'histoire de l'Amérique [de GeorgesBarthélémi Faribault] est une ouvre capitale dans le développement de l'intérêt pour l'histoire nationale au XIX $X^{e}$ siècle et pour l'histoire de la bibliographie canadienne.

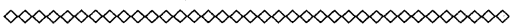

facile, dépendant, pour le choix des livres, des deux présidents de l'Assemblée et du Conseil législatif. À la question qu'il posait le 15 juillet 1887 : «Dois-je conclure que je ne puis rien acheter sans l'autorisation des deux orateurs? [...] Cela me met dans une position difficile», l'orateur du Conseil législatif, Pierre Boucher de la Bruère, lui répondait, le 18 du même mois:

"Je comprends qu'il n'est pas strictement nécessaire pour le bibliothécaire d'obtenir en toute occasion le consentement des deux orateurs, lorsqu'il s'agit de choses d'importance minime, mais dans le choix des livres et la direction de la Bibliothèque je me considère tenu de consulter l'orateur de l'Assemblée législative." (Archives du Séminaire de Trois-Rivières)

Après la défaite du gouvernement Mercier, les conservateurs, de retour au pouvoir sous la direction $\mathrm{du}$ premier ministre Charles-Eugène Boucher de Boucherville, souhaitaient des changements à la bibliothèque parlementaire. Aussi Narcisse-Eutrope Dionne, médecin et journaliste, lié au nouveau gouvernement, fut-il envoyé à Ottawa pour observer le fonctionnement de la Bibliothèque de la Chambre des communes. Ce geste fut perçu par LeMay comme une invitation à démissionner. Dans une chronique parue dans un journal, Arthur Buies commentait ce geste:

«[...] Telle encore cette hypocrisie de déléguer le docteur N.-E. Dionne à Ottawa "pour étudier le fonctionnement et l'administration de la bibliothèque fédérale". Mais, malheureux! vous donneriez tout au monde pour qu'il n'y eût pas de bibliothèque du tout à Québec. Etudier le fonctionnement de la bibliothèque fédérale! En voilà une par exemple qui vous étouffe. Mais qu'est-ce qu'il y a de si extraordinaire et de si mystérieux dans l'administration d'une bibliothèque pour qu'on charge un individu tout exprès d'aller étudier cela sur les lieux, dans une grande bibliothèque qui n'a aucune analogie avec la nôtre! [...] Comment, vous prétendez envoyer un délégué à Ottawa pour y étudier l'administration de la bibliothèque fédérale et vous n'avez rien de plus pressé que de destituer un écrivain qui a écrit précédemment des livres très estimés et très exacts sur votre propre pays! Et vous n'avez rien de 
plus pressé que de supprimer la bibliothèque des ouvriers de Saint-Roch!» (Buies, 1892)

À la fin de septembre 1892, Dionne devint bibliothécaire de l'Assemblée et pendant 20 ans travailla sans relâche à augmenter son prestige et son rayonnement. Dès son entrée en fonction, il intégra la riche collection de l'ex-premier ministre Chauveau que LeMay avait acquise. Il publia, en 1903, un catalogue qui recensait les 65000 volumes et les 17 ooobrochures formant alors la collection. En 1905, il publia également son important Inventaire chronologique des livres, brochures, journaux et revues publiés en langue française dans la province de Québec, depuis létablissement de l'imprimerie au Canada jusqu’à nos jours, 1764-1905, regroupant près de 9000 titres d'imprimés. Enfin, il réussit à faire accepter le projet de construction d'un nouvel édifice pour loger la Bibliothèque (Gallichan, 1984).

La direction de la Bibliothèque de la Législature est une fonction d'importance dans la société québécoise. En 1934, le gouvernement Taschereau nomma à ce poste Jean-Charles Harvey, journaliste et auteur du roman Les Demi-Civilisés. On s'en doute, les autorités ecclésiastiques de Québec virent d'un mauvais œil cette nomination et Harvey échoua finalement à la direction du Bureau des statistiques, tandis que le directeur de ce bureau, Georges-Émile Marquis, fut transféré à la Bibliothèque, où il occupa le poste de directeur de 1934 à 1952. Marquis fut remplacé en 1952 par Jean-Charles Bonenfant, qui donna à la Bibliothèque un essor et un lustre considérables. Secrétaire particulier du premier ministre Duplessis de 1937 à 1939, Bonenfant avait été muté lors de l'arrivée des libéraux au pouvoir. Il dirigera la Bibliothèque de 1952 à 1969. Sa culture encyclopédique, ses connaissances en droit parlementaire et constitutionnel, ses talents de communicateur et d'administrateur ont fait de Bonenfant un «bibliothécaire modèle» reconnu par l'ensemble de ses contemporains (Bernier, 2002). Entré en fonction par un simple concours de circonstances, sans aucune préparation professionnelle, Bonenfant a connu tous les arcanes de son institution et il en a fait une grande bibliothèque de référence. Il affirmait lui-même: "J'ai cherché, sans y réussir vraiment, à être le bibliothécaire idéal, celui qui, selon moi, maintient l'équilibre entre les connaissances techniques et la culture» (Bonenfant, 1977).

De 1893 à 1964, l'Université McGill n'eut à la tête de sa bibliothèque que trois directeurs - Charles Henry Gould (1893-1919), Gerhard R. Lomer (19201947) et Richard Pennington (1947-1964) -, à l'origine des excellentes collections développées au cours de ces trois quarts de siècle (McNally, 1988). Lors de son décès en 1919, Gould fut considéré comme le plus grand bibliothécaire canadien et l'un des plus grands d'Amérique du Nord (McNally, 1998). On ignore comment il obtint ce poste. Son expérience des affaires, ses études, son bagage culturel et le fait qu'il était presbytérien, tout comme le principal, sir John William Dawson, et le grand bienfaiteur de la Bibliothèque, Peter Redpath, jouèrent sans doute en sa faveur. Après sa nomination, il parcourut l'Europe et les États-Unis pendant un an pour s'initier au monde des bibliothèques. Responsable de la venue du congrès de l'American Library Association à Montréal, en 1900, Gould en fut le premier président canadien de 1908 à 1909. En 1912-1913, il devint le quatrième président de la Bibliographical Society of America. Il inaugura les premiers cours d'été en bibliothéconomie à McGill en 1904 et il invita son ami Melvil Dewey, de l'Université Columbia de New York, à y enseigner. Par-dessus tout, il fit passer les collections de McGill de 55000 à 180 ooo volumes et sut intéresser des mécènes comme John Redpath et William Macdonald à contribuer à l'enrichissement de la plus grosse bibliothèque universitaire canadienne. Avec Gould, la bibliothéconomie changea à la fois de siècle et de registre. Avec lui, les associations professionnelles naissantes commencèrent à influencer d'une manière appréciable le développement des bibliothèques.

$\mathrm{Au} \mathrm{XIX}^{\mathrm{e}}$ siècle, les bibliothèques sulpiciennes ne relèvent que les noms des responsables, notamment l'abbé Jacques-Victor Arraud pour l'CEuvre des bons livres, au cours de la décennie 1840, et l'abbé Louis Regourd, qui donna une nouvelle impulsion à la Bibliothèque et lança le Cabinet de lecture paroissial en 1857 (Lajeunesse, 1982). Il en est de même pour le pendant libéral, l'Institut canadien de Montréal. En fait, nous en savons plus sur les collections, sur la circulation des livres et des idées que sur les bibliothécaires qui $\mathrm{y}$ travaillèrent.

La bibliothèque du Fraser Institute, établie en 1870 à Burnside Hall à la suite d'un legs de l'homme d'affaires Hugh Fraser, acquit, en 1885, la collection de l'Institut canadien. Au début du XX $\mathrm{XX}^{\mathrm{e}}$ siècle, elle s'imposa comme la bibliothèque publique de langue anglaise de Montréal (Moodey, 1977). Son directeur, Pierre Boucher de Crèvecœur, donna à la Bibliothèque un relief remarquable. Français d'origine, entré en fonction en 1897 après avoir exercé plusieurs métiers et sans formation particulière, il dirigea la bibliothèque Fraser de 1901 jusquà son décès, en 1930. Cette bibliothèque "marqua le passage de la bibliothèque traditionnelle du XIX $X^{e}$ siècle à la bibliothèque moderne, dont les caractéristiques s'annoncèrent dans la dernière décennie du siècle» (Lamonde, 1979).

Pour sa part, Ægidius Fauteux apparaît, de 1912 à son décès en 1941, comme le bibliothécaire type au Québec, du moins dans sa partie francophone. Élève des Sulpiciens, avocat, journaliste, rédacteur en chef de La Presse, il est invité en 1912 à devenir conservateur de la Bibliothèque Saint-Sulpice, alors en construction. Après avoir rassemblé quelque 60 ooovolumes des bibliothèques de Sulpiciens, Fauteux fait de longs 
séjours aux États-Unis et en France de 1913 à 1914 pour y acquérir les volumes essentiels au démarrage de cette grande bibliothèque "publique» de référence, qui doit ouvrir ses portes en 1915 (Lassonde, 1987). Par le dynamisme dans le développement des collections, par la modernité des choix faits dans le traitement (système Dewey), par l'énergie consacrée à intégrer sa bibliothèque dans la vie culturelle montréalaise, Fauteux fit de la Bibliothèque Saint-Sulpice un lieu bien connu des intellectuels et des étudiants d'université. Après la fermeture de Saint-Sulpice en 1931, Fauteux consacra ses talents à la direction de la Bibliothèque municipale de Montréal. Il était de toutes les sociétés savantes et professionnelles (Société royale, les Dix, Société généalogique, membre de l'American Library Association depuis 1913, membre de la Quebec Library Association dès sa fondation en 1932); historien reconnu, il fut de plus, en 1937, cofondateur de l'École de bibliothécaires de l'Université de Montréal (Gallichan, 1996).

À la direction de la Bibliothèque municipale de Montréal, Fauteux avait été précédé par Hector Garneau (1916-1930), petit-fils de l'historien national, historien lui-même et responsable d'une édition critique de l'Histoire du Canada de son aïeul. Léo-Paul Desrosiers (1941-1952), journaliste, romancier et historien, et Jules Bazin (1952-1974), spécialiste en histoire de l'art, lui succédèrent. Ces nominations continuaient la tradition de bibliothécaires hommes de lettres. Lors de sa nomination, on a écrit que Garneau avait obtenu le poste à cause de "fortes aptitudes littéraires et d'une intellectualité spéciale» (La Presse, 1916). De la même façon, Desrosiers "réalisera une partie de son rêve en devenant conservateur de la Bibliothèque municipale» (Groulx, 1971; Gallichan, 1996b; Gervais, 1977).

\section{LES DÉBUTS DE LA PROFESSIONNALISATION}

À la fin du XIX ${ }^{\mathrm{e}}$ siècle, les bibliothèques américaines subirent des mutations profondes. Les bibliothécaires des États-Unis fondèrent en 1876 la première association professionnelle, l'American Library Association, et la première revue spécialisée dans le domaine, The Library Journal. En plus délaborer un système de classification décimale, Melvil Dewey fondait, en 1887, à l'Université Columbia de New York, la première école de bibliothéconomie, ce qui favorisa l'émergence de grandes bibliothèques universitaires et de nombreuses bibliothèques publiques. Le développement et le traitement des collections, la constitution de bibliographies et de références, de même que la gestion des bibliothèques exigeaient un personnel de plus en plus spécialisé. Au cours du premier tiers du $\mathrm{XX}^{\mathrm{e}}$ siècle, des dizaines d'écoles de bibliothéconomie furent donc créées aux États-Unis et l'Université de Chicago mit sur pied le premier programme de doctorat en 1928. À la suite de la publication du rapport Williamson, l'American Library Association normalisa la formation en bibliothéconomie en créant, au cours de la décennie 1920, un organisme d'agrément des écoles.

Ces événements eurent des répercussions au Canada et au Québec. Comme nous l'avons déjà mentionné, l'American Library Association tint son congrès annuel pour la première fois au Canada, à Montréal, en 1900 et l'Université McGill inaugura ses premiers cours d'été en 1904, avec la contribution de Melvil Dewey. Ces cours de bibliothéconomie seront offerts sur une base régulière à partir de 1927 et l'Université McGill sera la première à être agréée au Canada par l'American Library Association. Les premiers diplômes de baccalauréat en bibliothéconomie seront ainsi remis aux étudiants en 1931 (McNally, 1996). L'Université McGill offrit, à l'été 1932, une session d'études en langue française. Enfin, quelques bibliothécaires de langue française tels Ægidius Fauteux, Marie-Claire Daveluy et Hélène Grenier devinrent membres de la Quebec Library Association, qui fut fondée, en 1932, pour regrouper principalement les bibliothécaires de langue anglaise.

Marie-Claire Daveluy et Hélène Grenier, toutes deux diplômées de l'École de bibliothécaires de McGill, l'une dans les années 1920 et l'autre dans la décennie suivante, contribuèrent de façon importante à la structuration de la bibliothéconomie québécoise au cours des années 1930. En 1937, Marie-Claire Daveluy participa activement à la mise sur pied de l'École des bibliothécaires de l'Université de Montréal, conjointement avec Ægidius Fauteux et le père PaulAimé Martin. Trois ans plus tard, elle présentait dans la revue Culture les objectifs de la nouvelle École:

«Tout d'abord la formation de bibliothécaires de carrière, qu'il s'agisse d'institutions, publiques ou privées, de culture générale ou spécialisée [...] Le développement de la science de la bibliographie chez les nôtres; l'apparition de bibliographes-auteurs sérieux, compétents, laborieux. Notre indigence bibliographique est extrême. Sauf quelques travaux, qui nous empêchent de courber trop la tête, tout est à faire chez nous dans ce domaine. [...] Enfin, l'École de bibliothécaires veut faire connaître au moyen de catalogues bien rédigés les ressources de nos dépôts de livres déjà existants. » (Daveluy, 1940)

Et d'ajouter quelques années plus tard: «On ne simprovise pas plus bibliothécaire ou bibliographe que chimiste, linguiste ou théologien: il faut de la science, de la technique, de l'expérience.» Daveluy jouissait d'une grande autorité dans le milieu des bibliothèques et chez les intellectuels du Québec. Bibliothécaire à la Bibliothèque municipale de Montréal depuis 1917 et chef du catalogue, bibliographe, écrivaine pour la jeunesse, historienne, érudite, elle était à l'avant-scène 
de la vie culturelle montréalaise. Elle a participé d'une manière considérable à la rédaction du programme d'études de l'École en accordant à la bibliographie une place prédominante; pour elle, la bibliographie était le «vestibule de toute science» (Morisset, 1977). Hélène Grenier, directrice de la Bibliothèque des instituteurs et des bibliothèques scolaires à la Commission des écoles catholiques de Montréal, marqua aussi l'histoire de la bibliothéconomie du Québec pendant trois décennies. Grenier jugeait la carrière de bibliothécaire méconnue, la population s'en faisant une idée très approximative. Selon elle, cette profession requérait une instruction solide, une bonne érudition, l'amour des livres et surtout l'amour des humains; elle insistait sur le rôle social du bibliothécaire et sur la contribution de la bibliothèque publique à l'éducation postsecondaire (Grenier, 1937 et 1942). Aux États-Unis, la formation de bibliothécaires était assurée dans les écoles de bibliothéconomie des grandes universités, un modèle qu'elle croyait important de suivre.

À partir de 1945, l'École de bibliothécaires offrit un programme de baccalauréat aux diplômés du baccalauréat ès arts. Pendant ses 25 ans d'existence, l'École délivra 545 diplômes ou attestations, 41 diplômes techniques supérieurs (aux étudiants admis après un baccalauréat de rhétorique des collèges classiques) et 134 baccalauréats en bibliothéconomie; pourtant, elle ne fut jamais un département de l'Université de Montréal. Dépourvue d'un corps professoral régulier et de ressources, ses liens avec l'Université se résumaient à l'approbation des programmes d'étude par la Commission des études et à la délivrance des diplômes universitaires (Durand, 1977). L'École avait été créée pour former des techniciens au moment où le monde du livre, de l'édition et des bibliothèques commençait à ressentir des besoins en personnel spécialisé. Une proportion importante de la clientèle étudiante des premières années venait des communautés religieuses. Dans une lettre de septembre 1938, Ægidius Fauteux écrivait ainsi qu'une bonne partie de l'assistance se composait de prêtres et de religieuses que les communautés avaient délégués pour s'initier à l'art de tenir une bibliothèque; et en février 1945 LéoPaul Desrosiers informait le secrétaire de la province que $30 \%$ des étudiants étaient prêtres ou religieux (Savard, 1997). Il va de soi que les bibliothèques des communautés religieuses, des institutions d'enseignement et les bibliothèques paroissiales étaient visées au premier chef.

Cofondateur de l'École de bibliothécaires, le père Paul-Aimé Martin commença au tournant des années 1940 une longue et brillante carrière d'éditeur. Associé étroitement au monde des bibliothèques, il fonda en 1943 l'Association canadienne des bibliothèques d'institution, qui devint, en 1945, l'Association canadienne des bibliothèques catholiques, puis, en 1948, l'Association canadienne des bibliothécaires de langue française. Jusque dans les années 1950, tant les programmes de l'École que les activités de l'Association visaient la constitution d'une profession de bibliothécaires formés sous l'égide du catholicisme:

«L'École ne forme pas seulement des techniciens du livre; elle forme de vrais bibliothécaires catholiques. Il y a par exemple des cours sur l'Index et sur les bibliothèques paroissiales. D'ailleurs, tout l'enseignement est imprégné d'esprit chrétien.» (Bertrand, 1947)

Même au début des années 1960, Raymond Tanghe affirmait qu'en plus des cours traditionnels de classification et de catalogage, de bibliographie, de développement des collections et d'administration dispensés dans toutes les écoles l'enseignement de l'École des bibliothécaires «comprenait en outre des sujets propres au milieu canadien-français, tels que l'histoire de l'Église au Canada, l'histoire du livre au Canada, l'édition au Canada, la censure ecclésiastique et la législation sur le droit d'auteur" (Tanghe 1962). Les objectifs de l'Association étaient appuyés par les périodiques Mes fiches, La Revue des bibliothèques et Lectures, publiés chez Fides, de même que par le Service des bibliothèques de la maison Fides. Rappelons ici que Paul-Aimé Martin était justement le fondateur et le directeur de cette entreprise.

L'Association canadienne des bibliothécaires de langue française tint son premier congrès annuel en 1951 et publia un bulletin professionnel à partir de 1955. Un sociologue a retracé, pour les années 1951 à 1968, l'évolution des idées qui ont guidé l'action des bibliothécaires canadiens-français et déterminé les changements de leur profession (Gagnon, 1976). Il distingue deux périodes : la première, de 1951 à 1960, est dominée par l'humanisme chrétien; la seconde, de 1960 à 1968, est caractérisée par la montée de l'idéologie scientifique. Dès le premier congrès, l'archevêque de Montréal, $\mathrm{M}^{\mathrm{gr}}$ Léger, soutenait qu' «une bibliothèque, c'est un sanctuaire, c'est une vie. Un bibliothécaire doit aimer ce temple qui conserve la pensée de l'Homme». Le bibliothécaire se devait aussi d'être moraliste par sa fonction. Au congrès de 1953, on affirmait que «si nous avons une ouvre d'éducation à accomplir comme bibliothécaires, c'est bien avant tout cette éducation morale». D'ailleurs, à l'intention des responsables du développement des collections des bibliothèques, les revues bibliographiques de Fides ne se privaient pas d'établir des cotes morales allant des livres mauvais ou dangereux aux livres qui appelaient des réserves plus ou moins graves, jusqu'aux livres convenables pour les adultes (Martin, 1946).

\section{BIBLIOTHÉCAIRE, UNE VÉRITABLE CARRIÈRE}

À la fin des années 1960, une transformation radicale apparaît dans la vision que les bibliothé- 
caires ont d'eux-mêmes et de leur profession. Le grand protagoniste de ce changement est le jésuite Edmond Desrochers. Détenteur d'une maîtrise en bibliothéconomie de l'Université Columbia en 1951, Desrochers exerça en peu d'années une influence certaine dans l'Association canadienne des bibliothécaires de langue française. Très au fait des activités de l'American Library Association et de la Canadian Library Association, il fit de l'association québécoise le levier des transformations souhaitées. Dès 1952, il publia une brochure sur Le Rôle social des bibliothèques publiques (Desrochers, 1952). Principal rédacteur du Mémoire de l'Association à la Commission Tremblay, déposé en mars 1954, il constatait la grande pauvreté des bibliothèques du Québec et proposait des mesures pour y remédier. Ce mémoire contenait en réalité de grands principes fondamentaux qui seront repris par la législation québécoise sur les bibliothèques publiques et scolaires des années 1959-1960. En 1960, Desrochers livra un vigoureux plaidoyer en faveur de la création d'une véritable École de bibliothéconomie universitaire, à l'instar des autres écoles agréées de l'Amérique du Nord (Desrochers, 1960). L'Université de Montréal saisit le message et créa, en 1961, l'École de bibliothéconomie. Parmi les multiples interventions d'Edmond Desrochers, certaines concernent également les bibliothèques de collège (Desrochers, 1961) et les bibliothèques universitaires (Desrochers, 1965).

Bien qu'il ait joué un rôle déterminant, il serait réducteur de voir en Edmond Desrochers le seul instigateur d'une vision plus scientifique, plus séculière et plus continentale de la profession. Jean-Rémi Brault a montré avec brio dans ses récents mémoires l'effervescence qui animait le monde des bibliothèques de collège classique à la fin des années 1950 et au cours de la décennie 1960 (Brault, 2004). Que dire ainsi de la tâche qui attendait Gérard Martin et ses collègues $\mathrm{du}$ Service des bibliothèques publiques à la suite de la promulgation de la Loi sur les bibliothèques publiques en décembre 1959? Tout était à faire! Sur les quelque 7obibliothèques «publiques» recensées en 1960, la plupart fonctionnaient dans l'amateurisme le plus total: 58 d'entre elles n'avaient aucun personnel professionnel, les ressources financières étant si faibles qu'elles ne permettaient que le bénévolat. Inutile d'ajouter qu'aucune bibliothèque ne desservait alors la population rurale du Québec (Martin, 1977; Gagnon, 1985).

Au cours de la décennie 1960, le développement des véritables bibliothèques universitaires, de collège et publiques, la création de la Bibliothèque nationale du Québec (1967), de même que la formation des bibliothécaires à McGill et à la nouvelle École de bibliothéconomie amènent les bibliothécaires à se pencher, au cours de leurs congrès annuels, sur le caractère et l'exercice de leur profession. Au congrès annuel de 1961, le sociologue Jacques Lazure aborde les problèmes de la professionnalisation. Pour lui, «une fonction ne peut tailler sa place au soleil de la profession que dans la mesure où la société, en général, reconnaît et sanctionne son caractère social et professionnel» (Lazure, 1962). Le bibliothécaire, affirme-t-il, devra toujours considérer le contenu intellectuel du livre, non pas pour lui-même, mais dans sa relation d'instrument au service de l'homme. Il fait référence aux études d'Everett Hughes, le spécialiste américain de la sociologie des professions, pour qui la bibliothéconomie est une «emerging profession» qui tend de plus en plus vers le statut professionnel. Deux caractéristiques ressortent: la formation d'un corpus de science spécialisée qui nécessite de longues études appropriées et un idéal élevé de service à la collectivité humaine. Dans cette optique, le bibliothécaire est au service de la lecture, de la recherche, de linformation. Claude Ryan, invité à livrer ses commentaires lors du même congrès, émet quant à lui un constat sévère à l'égard de la pratique du bibliothécaire québécois (Ryan, 1962). Pour lui, la bibliothèque est un instrument et un facteur de progrès intellectuel. Le bibliothécaire a un rôle actif à jouer dans la vie et la production intellectuelle de son milieu, il doit être au service du public. En fait, il doit être à la fois un témoin, un serviteur et un artisan de la culture. Or, selon lui, le bibliothécaire québécois ne réussit pas à atteindre cet idéal.

L'Association canadienne des bibliothécaires de langue française (ACBLF) a joué un rôle de premier plan dans la professionnalisation du métier, même si "l'ACBLF avait été créée à une époque où la plupart des personnes qui portaient le titre de bibliothécaire ne l'étaient pas vraiment». En effet, selon Jean-Rémi Brault:

$$
\begin{aligned}
& \text { "Peu d'entre elles avaient étudié la biblio- } \\
& \text { théconomie. Rares étaient ceux ou celles qui } \\
& \text { avaient obtenu un diplôme dans cette disci- } \\
& \text { pline. Tout au plus, quelques-uns avaient par- } \\
& \text { ticipé à quelques jours de stage. La plupart } \\
& \text { étaient devenus "bibliothécaires" parce que } \\
& \text { les circonstances de la vie ou de la carrière les } \\
& \text { avaient entraînés à travailler dans une biblio- } \\
& \text { thèque.» (Brault. 2004) }
\end{aligned}
$$

Cela était vrai pour les décennies 1940 et 1950; ce ne l'était plus à partir des années 1960. Les congrès annuels de l'Association permettaient de débattre les enjeux de cette profession en développement (Léveillé, 1977). Au milieu des années 1960, à cause de l'arrivée des syndicats dans les bibliothèques et aussi d'un nouveau personnel, les bibliotechniciens, on discuta des moyens et des structures les plus appropriés pour faire avancer la professionnalisation du bibliothécaire au Québec. Le congrès de 1968 portait d'ailleurs sur les associations et les syndicats (Association..., 1969).

Un premier comité mixte -Quebec Library Association/Association canadienne des bibliothé- 
caires de langue française - avait été créé en 1965 pour étudier la situation juridique du bibliothécaire professionnel dans son milieu. Ce comité en vint à la conclusion que seul un un ordre professionnel pouvait donner une définition inscrite dans un texte de loi et ainsi couper court aux discussions. Un projet de loi en ce sens fut rédigé et approuvé par les deux associations à l'été 1966 (Lefebvre, 1977). Le 30 mai 1969, l'Assemblée nationale du Québec sanctionnait la loi 175 constituant la Corporation des bibliothécaires professionnels du Québec. La création de la Corporation visait d'abord et avant tout à renforcer le statut professionnel du bibliothécaire. L'objectif ne fut rempli qu'en partie puisque, au début des années 1970, à la suite des travaux de la Commission Castonguay-Nepveu, le Code des professions fut revu en profondeur et la Corporation des bibliothécaires professionnels ne fut pas incluse dans le Code des professions, à la suite de la loi 250. La Corporation des bibliothécaires professionnels du Québec n'est toujours pas un ordre professionnel de plein droit, mais bien un ordre professionnel «à titre réservé».

On l'a vu, l'École de bibliothéconomie de l'Université de Montréal avait été créée pour développer la recherche dans la discipline et dispenser une formation égale à celles des meilleures écoles du Canada et des États-Unis. Le programme de baccalauréat lancé dans les années 1960 fut remplacé en 1970 par une maitrise comme seul programme professionnel, à l'instar de la pratique nord-américaine. Un programme de doctorat fut mis en place en 1997 et la première docteure soutint sa thèse six ans plus tard. De 1962 à 2003, l'École a décerné 437 baccalauréats (1962-1971) et 1830 maitrises (1971-2003) (Lajeunesse, 2002). Son enseignement s'est étendu, à partir des années 1980, aux sciences de l'information et à l'archivistique, l'objet d'étude étant dorénavant défini comme l'information enregistrée dans des documents. En 1984, l'institution prit le nom d'École de bibliothéconomie et des sciences de l'information.

En 1973, à la suite de la création de la Corporation des bibliothécaires professionnels du Québec, l'Association canadienne des bibliothécaires de langue française, qui existait depuis les années 1940 et recrutait $90 \%$ de ses membres au Québec, devient l'Association pour l'avancement des sciences et des technologies de la documentation (ASTED). Elle abandonnait ainsi sa charte fédérale pour une charte québécoise et visait à regrouper bibliothécaires, techniciens en documentation et tous ceux qui s'intéressaient à la promotion des bibliothèques et de la documentation.

Le bel ouvrage de Jean-Rémi Brault paru en 2004 constitue une introduction commode en même temps qu'il livre une vision articulée de l'évolution des bibliothèques québécoises pour la période allant de la fin des années 1950 à l'actuel millénaire. Ces premiers mémoires d'un bibliothécaire québécois sont en plus le témoignage d'un acteur profondément engagé dans les transformations qui ont été accomplies. Ils témoignent avec éloquence de la modernité de la bibliothèque et du bibliothécaire québécois d'aujourd'hui.

\section{CONCLUSION}

$\mathrm{Au}$ Québec, comme dans toutes les sociétés du livre, des bibliothèques ont rassemblé des collections à l'usage des législateurs, des étudiants et des professeurs et de la population. Des bibliothécaires, gens de lettres, intellectuels reconnus pour leur érudition ont animé et dirigé pendant longtemps ces foyers culturels. Plusieurs de ces bibliothécaires "improvisés" sont devenus des bibliothécaires de grande stature.

$\mathrm{Au}$ cours de la décennie 1930, des changements s'opèrent dans la pratique de la profession. En 1932 est créée la Quebec Library Association. Après l'Université McGill, qui dispense un baccalauréat depuis 1927, l'École de bibliothécaires ouvre ses portes en 1937. Une association de bibliothécaires francophones voit le jour en 1943 et tient son premier congrès annuel en 1951, quatre ans avant de lancer son bulletin trimestriel. Tous ces événements témoignent de la structuration de la profession de bibliothécaire.

Avec les années 1960 émerge la volonté de doter cette profession d'un caractère scientifique. À la suite d'une première législation, une politique de lecture publique est mise en place. Des mutations profondes dans la formation dans les collèges exigent la création de véritables bibliothèques de collège scientifiquement organisées. Le développement fulgurant des études supérieures et de la recherche requiert des bibliothèques universitaires autrement mieux garnies. Les congrès professionnels de l'ACBLF reflètent les exigences de la société québécoise relativement aux bibliothèques et aux besoins de leurs membres. À l'image de la société, on assiste à une sécularisation de la vie associative: le patronage de Notre-Dame-du-Livre, qui était celui de l'ACBLF depuis 1948, sans doute une particularité bien québécoise, est abandonné. L'École de bibliothéconomie de l'Université de Montréal s'est donné, pour sa part, lors de sa fondation en 1961, l'objectif de former les bibliothécaires professionnels dont les nouvelles bibliothèques avaient besoin. La création de la Corporation des bibliothécaires professionnels du Québec en 1969 est une manifestation de la maturité du groupe professionnel des bibliothécaires. La transformation de l'ACBLF en l'ASTED en 1973 tient compte de la nouvelle donne dans le monde de la documentation. La réflexion et la recherche dans le domaine se trouvent dans les deux revues, Argus, de la Corporation, et Documentation et Bibliothèques, de I'ASTED; toutes deux ont des visées scientifiques.

Ce survol de l'évolution de la profession de bibliothécaire au Québec montre bien les changements intervenus en ce qui concerne le rôle social 
de ce professionnel. Il apparait d'abord comme un homme de lettres, estimé certes, mais exerçant un pouvoir limité, soumis qu'il était au pouvoir politique. La fonction de bibliothécaire du Parlement en est l'illustration la plus patente. Puis, à partir du milieu du XIX ${ }^{\mathrm{e}}$ siècle, à la suite de la montée en puissance de l'Église et de son emprise sur le monde du livre et des bibliothèques, le bibliothécaire fut, pour un siècle, en milieu francophone, sous la tutelle du pouvoir religieux et il devint en quelque sorte gardien de la morale. Enfin, au tournant des années 1960, suivant en cela les mutations profondes de la société québécoise, le bibliothécaire put se mettre au service de la culture, puis à celui de l'information; il se perçoit et se définit comme un véritable professionnel. Le bibliothécaire n'a pas vécu et n'a pas œuvré en dehors des grands courants qui ont dominé la société, mais il a souvent représenté la culture et la liberté dans un milieu qui ne mettait pas ces valeurs au premier plan. $\odot$

\section{SOURCES CONSULTÉES}

Archives du Séminaire de Trois-Rivières. Fonds Pierre Boucher de la Bruère. Correspondance.

Association canadienne des bibliothécaires de langue française, 1954. Mémoire de l'Association [...] présenté à la Commission royale d'enquête sur les problèmes constitutionnels, à Montréal, le jeudi 18 mars 1954, Montréal, 16p.

Audet, Louis-Philippe. 1971. Histoire de l'enseignement au Québec, vol. 1 : 1608-1840, Montréal, Holt, Rinehart et Winston, 432 p.

Beaulieu, André. 1972. La Première Bibliothèque canadienne, 1632180o/The First Canadian Library, 1632-180o. Ottawa, Bibliothèque nationale du Canada, $62 \mathrm{p}$.

Bernier, Gaston. 2002. «Jean-Charles Bonenfant, un bibliothécaire modèle", Documentation et Bibliothèques, vol. 48, p. 51-54.

Bertrand, Théophile. 1947. «Dixième anniversaire de fondation de l'École de bibliothécaires ", Lectures, vol. 2, nº 6, p. 216-217.

Bonenfant, Jean-Charles. 1977. «Préface » in Georges-A. Chartrand, Livre, Bibliothèque et Culture québécoise, Montréal, ASTED, p. 9-11.

Brault, Jean-Rémi. 2004. Regard sur l'évolution des bibliothèques québécoises : récit d'un itinéraire professionnel, Montréal, Éditions ASTED, $282 \mathrm{p}$.

Buies, Arthur. 1892. «Chronique», La Patrie, 3o juillet 1892, p.1.

Choquette, Robert. 1959. Textes, choisis et présentés par André Melançon. Montréal, Fides, 95 p.

Daveluy, Marie-Claire. 1940. «L'École de bibliothécaires de l'Université de Montréal», Culture, vol. 1, p.13-18.

Desrochers, Edmond. 1952. Le Rôle social des bibliothèques publiques, Montréal, Bellarmin, $32 \mathrm{p}$.

. 1960. «Une école professionnelle de bibliothécaires", Relations, $2 \mathrm{O}^{\mathrm{e}}$ année, $\mathrm{n}^{\circ} 236$, p. 214-215. Cet article a paru en anglais dans Feliciter, n ${ }^{\circ} 6$ (janvier 1961), p.31-33.

Desrochers, Edmond. 1961. Programme pour une bibliothèque collégiale, Montréal, ACBLF, $82 \mathrm{p}$.

. 1965. "Aurons-nous d'authentiques bibliothèques universitaires?", Relations, $25^{\mathrm{e}}$ année, no 298, p. 295-297.

Drolet, Antonio. 1961. «La bibliothèque du Collège des Jésuites, 1632-1799. Essai de reconstitution", Revue d'histoire de l'Amérique française, vol. $14, \mathrm{n}^{\circ} 4, \mathrm{p} .487-54.4$.
Durand. Marielle. 1977. «L'École de bibliothécaires de l'Université de Montréal ", in Georges-A. Chartrand. Livre, Bibliothèque et Culture québécoise, Montréal, ASTED, p. 485-507.

Falardeau, Jean-Charles. 1972. «Étienne Parent», Dictionnaire biographique du Canada, Québec, Presses de l'Université Laval, vol. X, p. 633-641.

Faribault, Georges-Barthélemi. 1837. Catalogue d'ouvrages sur l'histoire de l'Amérique et en particulier sur celle du Canada, de la Louisiane, de l'Acadie, et d'autres lieux ci-devant connus sous le nom de Nouvelle-France, Québec, Cowan, 207 p.

Filion, Paul-Émile. 1977. «La première bibliothèque canadienne : le Collège des Jésuites de Québec : historique et contribution à l'inventaire des fonds » in Georges-A. Chartrand. Livre, Bibliothèque et Culture québécoise, Montréal, ASTED, p. 273-298.

Gagnon, Gilbert. 1976. «Les idéologies des bibliothécaires canadiensfrançais (1951-1968)», Documentation et Bibliothèques, vol. 22, $\mathrm{n}^{\circ} 4$, p. 169-179.

. 1985. «La politique d'aide au développement des bibliothèques publiques au Québec (1960-1985) », Documentation et Bibliothèques, vol. $31, \mathrm{n}^{\circ}$ 1, p.9-25.

Galarneau, Claude. 1980. «Dudevant, Arnaud-Germain», Dictionnaire biographique du Canada, Québec, Presses de l'Université Laval, vol. VI, p. 726-728.

Gervais, Claire. 1977. «Les bibliothécaires canadiens-français écrivains de 1850 à 1975 ", in Georges-A. Chartrand. Livre, Bibliothèque et Culture québécoise, Montréal, ASTED, p. 425462.

Gallichan, Gilles. 1984. «Un pionnier de la bibliographie québécoise : Narcisse-Eutrope Dionne, bibliothécaire de la Législature (1892-1912)», Cahiers du livre ancien du Canada français, vol. 1, p. 4-16.

1988. «Les bibliothécaires et leur conscience historique », Documentation et Bibliothèques, vol. $34, \mathrm{n}^{\circ} 3$ (juillet-septembre 1988), p.99-101.

1991. Livres et Politique au Bas-Canada, 1791-1849. Sillery, Septentrion, $519 \mathrm{p}$.

. 1996a. «Ægidius Fauteux, l’intellectuel généreux (18761941)», Cahier des Dix, $\mathrm{n}^{\circ}$ 51, p. 55-61.

. 1996b. «Léo-Paul Desrosiers, la vocation littéraire (1896-1967) ", Cahier des Dix, n ${ }^{\circ}$ 51, p. 61-69.

Grenier, Hélène. 1937. «La bibliothèque publique et l'éducation postsecondaire», Revue dominicaine, vol. 43, p. 308-311.

. 1942. «Voici le... bibliothécaire», L’Action universitaire, vol. IX, $\mathrm{n}^{\circ} 4$, p. 6-9.

Groulx, Lionel. 1971. Mes mémoires, tome 2 (1920-1928), Montréal, Fides, $418 \mathrm{p}$.

Lajeunesse, Marcel. 1982. Les Sulpiciens et la Vie culturelle à Montréal au XIX siècle, Montréal, Fides, 280 p.

, Marcel. 2002. «L'École de bibliothéconomie et des sciences de l'information (EBSI) de l'Université de Montréal, 1961-2003: quatre décennies de formation des spécialistes de l'information au Québec et au Canada français », Documentation et Bibliothèques, vol. $48, \mathrm{n}^{\circ}$ 4, p.125-135.

2004. «Le livre en Nouvelle-France et au début du Régime britannique $\left(17^{\mathrm{e}}-18^{\mathrm{e}}\right.$ siècles)», Revue française d'histoire du livre.

Lamonde, Yvan. 1979. Les Bibliothèques des collectivités à Montréal (17 $7^{e}-19^{e}$ siècles). Sources et problèmes. Montréal, Bibliothèque nationale du Québec, $139 \mathrm{p}$.

Lassonde, Jean-René. 1987. La Bibliothèque Saint-Sulpice, 1910-1931. $2^{\mathrm{e}}$ éd. Montréal, Bibliothèque nationale du Québec, $401 \mathrm{p}$.

Lazure, Jacques. 1962. "Aspects sociologiques de la profession de bibliothécaire », in La Profession de bibliothécaire. Rapport des 
travaux du $17^{e}$ Congrès, Montréal, 7-9octobre 1961, Montréal, ACBLF, p. 9-24.

Lefebvre, Louise. 1977. «La Corporation des bibliothécaires professionnels du Québec", in Georges-A. Chartrand. Livre, Bibliothèque et Culture québécoise, Montréal, ASTED, p. 801-812.

Léveillé, Jean-M. Bernard. 1977. «Les bibliothécaires canadiensfrançais et leurs 32 ans de vie en associations (ACBI-ACBLFASTED) ", in Georges-A. Chartrand. Livre, Bibliothèque et Culture québécoise, Montréal, ASTED, p. 699-723.

Martin, Gérard. 1977. «La Direction des bibliothèques publiques du Québec », in Georges-A. Chartrand. Livre, Bibliothèque et Culture québécoise, Montréal, ASTED, p. 651-664.

Martin, Paul-Aimé. 1946. «L'esprit de nos critiques et nos cotes morales", Lectures, vol. 1, p.1, p. 8-12.

McNally, Peter F. 1988. «Scholar librarians : Gould, Lomer and Pennington», Fontanus, vol. 1, p. 95-104.

1998. «Fanfares and celebrations: Anniversaries in Canadian graduate education for library and information studies ", in Peter F. McNally. Readings in Canadian Library History 2, Ottawa, Canadian Library Association, p. 39-56.

. 1998. "Gould, Charles Henry», Dictionnaire biographique du Canada, vol. XIV, p. 465-467.

Moodey, Edgar C. 1977. The Fraser-Hickson Library. An Informal History. Londres, Clive Bingley, 224 p.

Moledina, Sheza. 2004. «L'histoire des bibliothécaires», Bulletin des bibliothèques de France, t. $49, \mathrm{n}^{\circ}$ 3, p.100-101.
Morin, Paul. 2000. Euvres poétiques complètes, Édition critique par Jacques Michon, Montréal, Presses de l'Université de Montréal, $635 \mathrm{p}$.

Morisset, Auguste-Marie. 1977. «Marie-Claire Daveluy, bibliothécaire, bibliographe, écrivaine», in Georges-A. Chartrand, éd., Livre, Bibliothèque et Culture québécoise, Montréal, ASTED, p. 405-4.23.

Pariseau, Claude. 1972. La Bibliothèque du Collège des Jésuites, Montréal, McGill University Graduate School of Library Science, 24 p.

Pellerin, Maurice. 1998. «LeMay, Pamphile», Dictionnaire biographique du Canada, Québec, Presses de l'Université Laval, vol. XIV, p. 700-702.

et Gilles Gallichan. 1987. Pamphile LeMay, écrivain et bibliothécaire, Montréal, Bibliothèque nationale du Québec, $147 \mathrm{p}$.

Ryan, Claude. 1962. «Bibliothèques et bibliothécaires canadiensfrançais : réflexions d'un lecteur", in La Profession de bibliothécaire. Rapport des travaux du $17^{e}$ Congrès, Montréal, 7-9 octobre 1961, Montréal, ACBLF, p. 86-95.

Savard, Réjean. 1997. «Le discours sur la lecture et l'évolution des bibliothèques publiques au Québec de 1850 à 1950 », Argus, vol. $26, \mathrm{n}^{\circ} 2$, p. $19-27$.

Tanghe, Raymond. 1962. L'École de bibliothécaires de l'Université de Montréal, 1937-1962, Montréal, Fides, 69 p.

Thériault, Yvon. 1987. «Romain, François», Dictionnaire biographique du Canada, Québec, Presses de l'Université Laval, vol. VI, p. 726-728. 